\title{
Detection of Lung Nodules in Chest Radiographs Using Wiener Filter and D-CNN Model
}

\author{
Muhammad Zubair \\ Department of Electronics and Communication Engineering \\ Prasad V. Potluri Siddhartha Institute of Technology \\ Vijayawada, Andhra Pradesh, India \\ muhammadzub17@gmail.com
}

\author{
Antariksh Tiwari \\ Department of Multimedia Communication and Design \\ Central Institute of Technology \\ Kokrajhar, Assam, India \\ b18mcd005@cit.ac.in
}

\author{
Tanisha Banik \\ Department of Computer Science Engineering \\ Kalinga Institute of Industrial Technology \\ Bhubaneswar, Odisha, India \\ 26tanishabanik@gmail.com
}

\author{
Umesh Kumar Naik M \\ Department of Electronics and Communication Engineering \\ Prasad V. Potluri Siddhartha Institute of Technology \\ Vijayawada, Andhra Pradesh, India \\ umeshkumar.mudavath@gmail.com
}

\begin{abstract}
The automatic and accurate detection of lung tumours or nodules plays a vital role in diagnosing lung cancer. Radiologist have to study multiple chest $x$-rays to diagnose lung cancer. Since it is a very tedious and time-consuming task, lots of automatic nodule detection and classification techniques based on deep learning have been proposed in recent years. As the previous methods completely rely on deep networks, it increases the computational complexity of the nodule detection process, so in this work, we have proposed a modified wiener filter to remove the adaptive noise from the Chest X-Ray (CXR) images which has not been attempted by any researcher, further to make nodules clearer and contrasting we have enhanced the filtered CXR image by using adaptive histogram equalization (AHE). Later, we have performed classification using the simplified VGG network for Deep Convolutional Neural Network (D-CNN) model to detect and classify the lung nodules from CXR images. As a result, we got an accuracy of $92 \%$ in training and $94 \%$ on validation, which is then compared with the latest state-of-art works to prove the novelty of our proposed method. The dataset used in our study is taken from the Japan Society of Radiological Technology (JSRT). Finally, we have introduced a Computer Interface (CI) through which the proposed work can be implemented in real-time nodule detection applications in the near future.

Index Terms-Lung nodules, Chest X-rays (CXR), Wiener filter, Adaptive Histogram Equalization (AHE), Simplified VGG network, Deep Convolutional Neural Network (D-CNN), Computer Interface (CI)
\end{abstract}

\section{INTRODUCTION}

In 1895 [1] Wilhelm Conard Röntgen has discovered the Xrays, which drastically altered the field of medical diagnosis. At present, X-rays are replaced by ample of other medical imaging techniques such as computed tomography (CT) and magnetic resonance imaging (MRI). However, this is surely not the situation for the chest radiography or chest X-ray (CXR). Despite what might be expected, the conventional chest study is still by a long shot the most widely recognized kind of radiological method, making up, at any rate, $33 \%$ of all tests in a typical radiology department. The basic radiographic image of the malignant nodule is shown in Fig. 1.

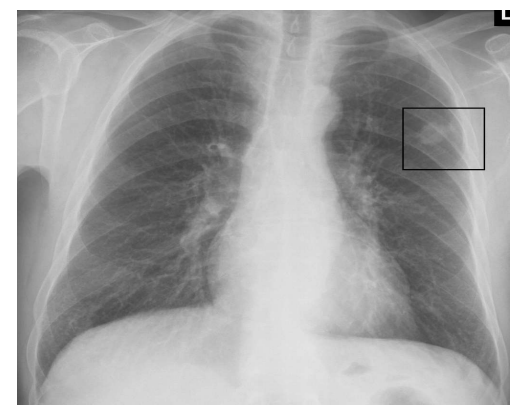

Fig. 1. Chest X-ray (CXR) containing malignant nodule

Though CT scan is more expensive and is not commonly available at local hospitals, CXR's have it's own upper hand in diagnosing the clinical lung cancers. So, detection of lung nodules automatically by computer-aided diagnosis (CAD) can ease the task of radiologists from manually examining the CXR's.

In recent years, due to plenty of CXR's, deep learning (DL) algorithms have emerged into a major part in analysing the CXR images [2] [3]- [4]. Wang [5] utilizes a feebly regulated multi-label image classification and illness confinement framework to distinguish the regularly happening thoracic infections. In [6] Irvin tried different things with different existing profound designs and ways to deal with the vulnerability in the manual labels to classify the 12 ordinarily happening pathologies in their CXR dataset. Different examinations have investigated the materialness of breaking down such datasets utilizing deep learning to help with radiological imaging analysis. In [7] Taylor utilized deep learning to identify pneumothorax in CXR images and received an accuracy of 94\%. Yao [8] utilized long short-term memory (LSTM) to use interdependencies among target names in CXR images. Similarly, Zech [9] examined the generalizability of deep learning models with respect to images gathered at various 


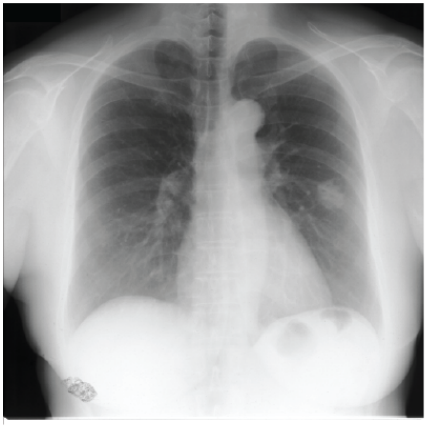

(a)

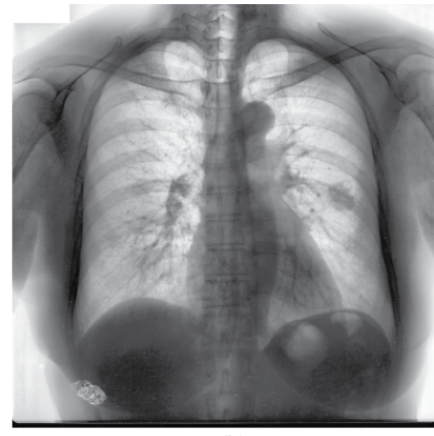

(b)
Fig. 2. Preprocessing output (a) Original Chest X-ray image, (b) Adaptive histogram equalization output

clinics to recognize pneumonia and acquired an accuracy of 93\% when the model was prepared on images taken from a similar location, and altogether got lower accuracy of $75 \%$ when prepared on images from different locations.

So to overcome the problem of computational complexity and to acquire high accuracy in detection and classification of lung nodules, we have proposed a technique which uses modified wiener filter and AHE to enhance the nodules in CXR image. And for classification between benign and malignant nodules we have used simplified VGG network for D-CNN model which clearly gave promising results when compared with the present state-of-art works.

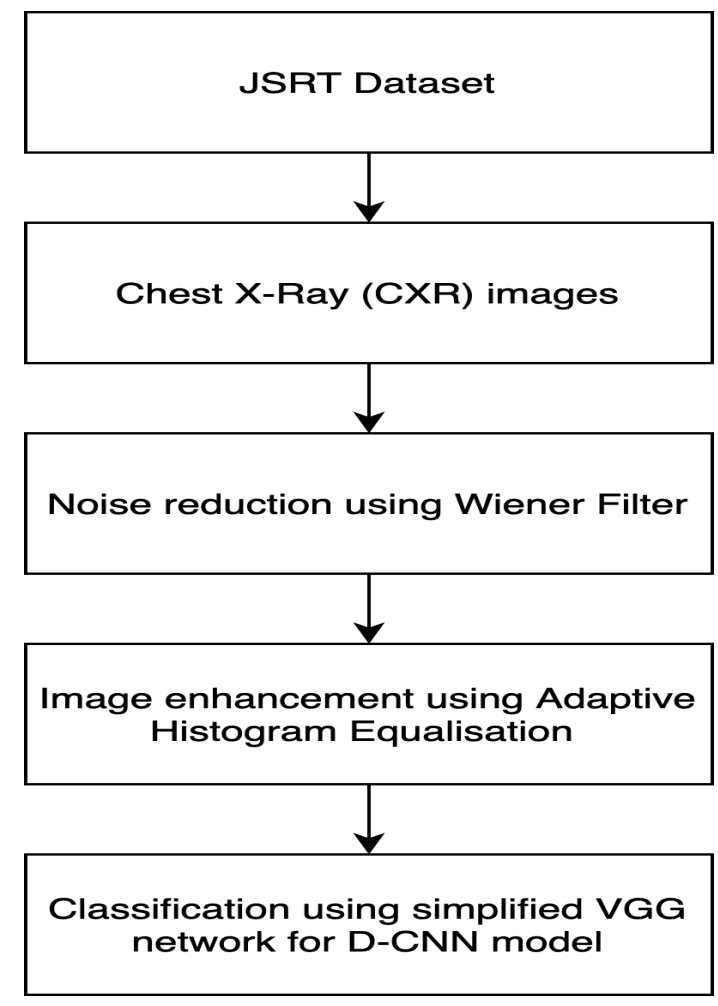

Fig. 3. Flowchart of the proposed work

\section{Proposed Methodology: Detection of Lung Nodules ANd Classification USING D-CNN}

\section{A. Data Description}

For this study we have used the dataset recorded at Japanese Society of Radiological Technology (JSRT) [10]. It is a standard digital image database containing radiographic images with and without chest lung nodules. This dataset consist of 154 images with lung nodules and 93 non-nodule images. Each image is of high resolution with $2048 \times 2048$ matrix size and with a pixel size of $0.175 \mathrm{~mm}$. This dataset can be used for training and testing the deep learning model for the diagnosis of lung cancer.

\section{B. Pre-processing using Wiener filter}

To prepare the CXR images for classification, we need to reduce the noise and enhance the images so that the nodules are identified by the deep learning model. Previously, for doing the preprocessing part lots of frameworks have been proposed such as U-Net and mask R-CNN. The main drawback of these methods is that they require high GPU computers to implement the networks and moreover they are not feasible to apply on radiographic images as they are widely used in computed tomography (CT) images.

To overcome these limitations we have proposed a simple and efficient preprocessing technique to enhance the lung nodules. In this work, we have used modified wiener filter to remove the adaptive noise present over CXR images [11]. Wiener filter uses the least square and its an universal approach to reduce the mean square error in the images. It is often defined as an optimal and non-linear filter. Wiener filter is designed as given in the equation below:

$$
G=\frac{\mathbb{H}^{*}}{|\mathrm{H}|^{2}+1 / S N R}
$$

Where, $\mathrm{H}$ is degradation function and $\mathbb{H}^{*}$ is its complex conjugate.

\section{Nodule enhancement using adaptive histogram equaliza- tion}

After performing the wiener filtration the CXR image loses its brightness and clarity. So to enhance and de-haze the image we have applied adaptive histogram equalization. After this process lung nodules were clearly visible and the outputs are further forwarded for classification using D-CNN model. The output of the preprocessing part is shown in Fig. 2 and flow chart of our proposed work is shown in Fig. 3.

\section{VGG architecture for D-CNN model}

In the proposed method, we use the simplified VGG network as the architecture for D-CNN model, which has one layer less than the original VGG network. It comprises of 6 convolution layers excluding the first with the inputs and 3 max-pooling layers stacked on each other alternatively. Each of the 11 convolution layers has 16,32 and 64 filters respectively with a common kernel of size $3 \times 3$. All the 4 max-pooling layers have a window of size $2 \times 2$ and a Global Max Pooling layer before 


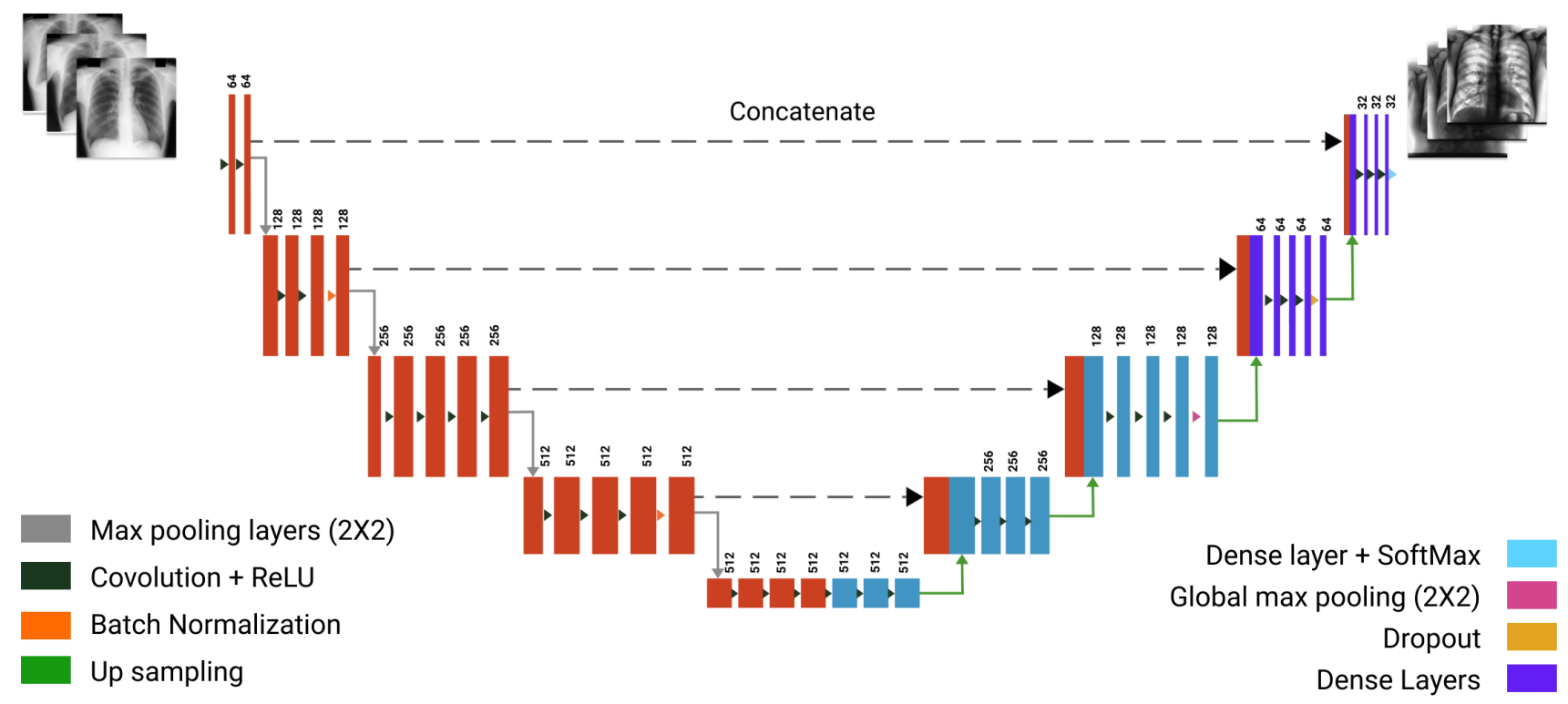

Fig. 4. Simplified VGG network

the first dense layer. The fully connected layer have 1 hidden layer with 32 nodes and 1 output layer. Finally, the output layer has 2 classes which represent Benign and Malignant nodules.

\section{EXPERIMENTAL RESULTS}

Initially, the CXR images were sorted into different folders based on the presence of nodules. Since all the X-ray images contain a considerable amount of noise, in our work, we have applied wiener filter of size [5,5] to suppress the undesired noise. While removing the noise in the image, few pixels related to nodule also gets hazed, So, in order to enhance the visibility of nodule we have applied adaptive histogram equalization on the filtered image which is then forwarded to the VGG network for classification.

\section{A. Classification using D-CNN}

The CNN architecture comprises three layers, namely Convolutional Layer (CL), Max Pooling Layer (MPL), and Fully Connected Layer (FCL). In the provided D-CNN network architecture; We have used $6 \mathrm{CL}$ excluding the first layer with the inputs of kernel size 3,4 MPL with window size [2,2], 1 Global Max Pooling Layer and 2 FCL with 32 and 3 nodes respectively.

The network is trained to detect important features of lung cancer such as nodules. The output is then passed through a non-linear activation function known as a rectified linear unit (ReLU). Later, the obtained image is passed through the MPL to down-sample the image for accelerating the training process and to reduce the amount of memory consumed by the network. A detailed architecture of the proposed work is explained in the block diagram shown in Fig. 3.

\section{FUTURE APPLICATION IN REAL WORLD}

In this section, we have designed how the concept can work in the real world. In the following diagram Fig. 6, the patient's scan will take place to get the CXR data of the lungs. This data will be sent through the proposed algorithm to perform the task of pre-processing and classification. The system's analyzed result is then being accessed through a computer interface and shown to the user. This method reduces the time for initial diagnosis, which can be proved vital for patient's treatment. Designing the whole application for it will be the future scope. The application will output if the lung nodule is cancerous or not based on the classification i.e., malignant and benign. This will help people by instantaneously giving the diagnosed results on their hands and not depending on further radiography procedures. It will enable patients to quickly consult a doctor and go for further medical procedures without delay. In hospitals, this can allow them to prioritize patients for medical treatments. Those who have more chances of developing cancer cells can immediately be taken care of, and others can follow up the rest.

\section{DISCUSSION}

In this study, we have compared the performance of our proposed method with existing approaches for the classification of LN or non-LN from the CXR's. In the recent study, M. S. Majdi et al. [12] have proposed a deep learning network for classification of pulmonary nodules and cardiomegaly based on ROC analysis in CXR images. They obtained a moderate area under the curve (AUC) of 0.73 for nodule classification and high AUC (0.91) for cardiomegaly classification.

Moreover, A. Siamak et al. [13] have used the VGG-16 architecture to develop a deep convolutional neural network 


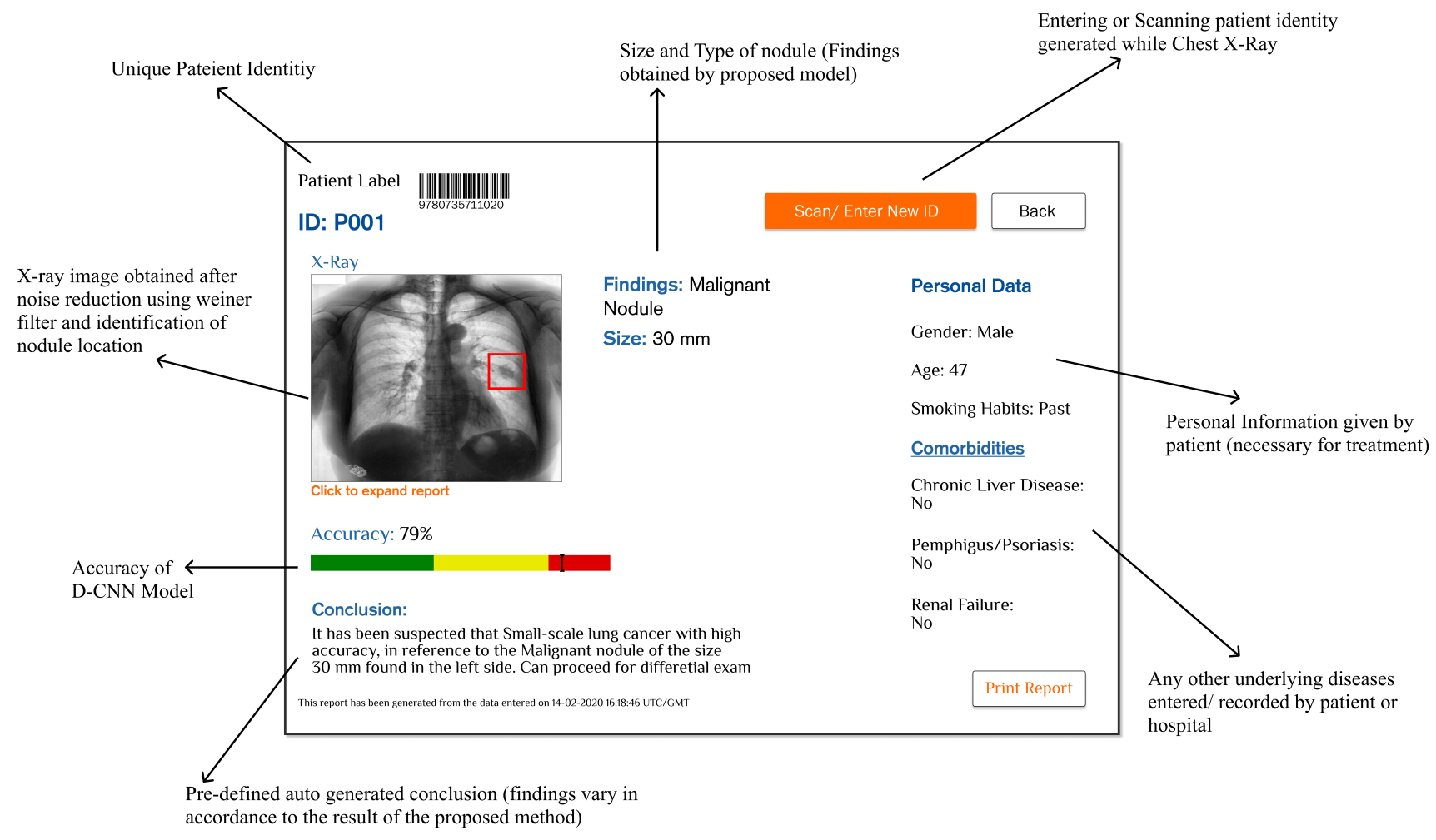

Fig. 5. Proposed computer interface for proposed method

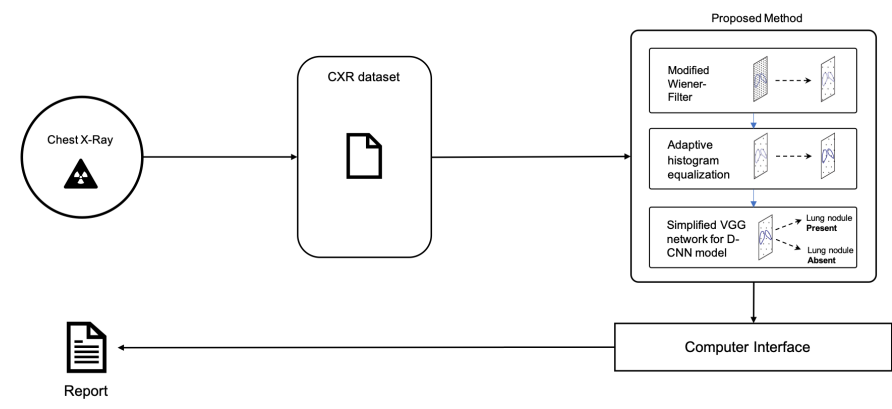

Fig. 6. Flow chart of the application design

(D-CNN) for the classification of 14 different diseases. The DCNN classifier has obtained an accuracy of $89.06 \%$ for 2749 nodule samples. Similarly, P Rajpurkar [14] have developed an algorithm that can detect pneumonia from CXR's at a level exceeding practising radiologists. The algorithm, CheXNet, is a 121-layer convolutional neural network trained on CheXNet to detect all 14 diseases in CXR's. Moreover, L. Utkin et al. [15] have implemented a lung cancer CADx system based on applying the ensemble of TrNNs. They have obtained an accuracy of $63.8 \%$ by the ensemble of random forests and accuracy of $91 \%$ by the ensemble of TrNNs respectively.

All the works mentioned above presents complex algorithms with less accuracies in detection and classification of lung nodules in CXR images. Moreover, previous papers didn't emphasise the importance of design interface which is a key aspect to implement the proposed work in real life clinical use. In our work we proposed a new algorithm which combines wiener filter with the VGG networkl to detect and classify lung nodules with less computational complexity and high accuracy of $92 \%$. The plots representing our training and validation accuracy are shown in Fig. 6.

The parameters used for evaluating the classifiers in this study are sensitivity, and accuracy as can be calculated by equation (2) to (3).

$$
\begin{gathered}
\text { Sensitivity }=\frac{\mathrm{TP}}{\mathrm{TP}+\mathrm{FN}} \\
\text { Accuracy }=\frac{\mathrm{TP}+\mathrm{TN}}{\mathrm{TP}+\mathrm{FP}+\mathrm{TN}+\mathrm{FN}}
\end{gathered}
$$

where, TP=True Positive, TN=True Negative, FN=False Negative, FP=False Positive

The ROC curve which is plotted with TPR against FPR is shown in Fig. 7. 


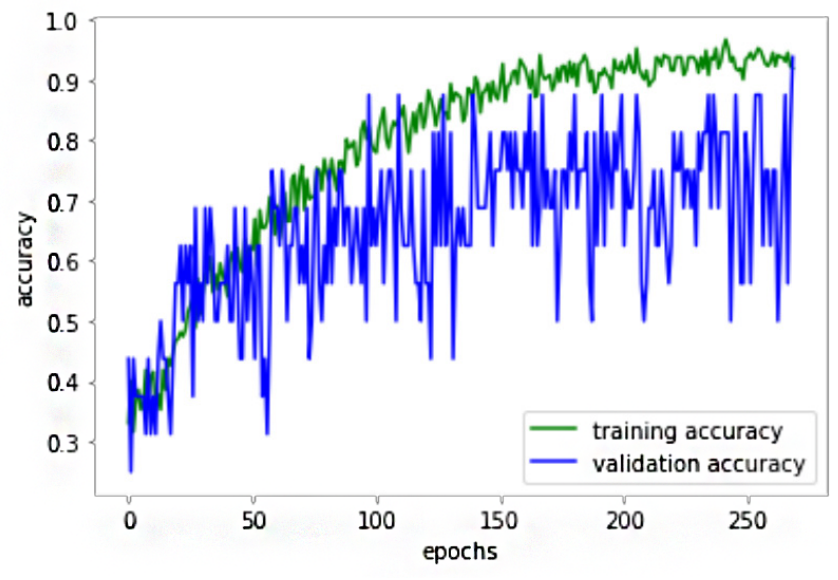

Fig. 7. Graphical representation of training and validation accuracy

TABLE I

COMPARISON OF ACCURACY AND RECALL BETWEEN VARIOUS CLASSIFICATION TECHNIQUES AND PROPOSED TECHNIQUE.

\begin{tabular}{|l|l|l|}
\hline \multirow{2}{*}{$\begin{array}{l}\text { Classification } \\
\text { techniques }\end{array}$} & \multicolumn{2}{|c|}{ Lung nodule classification } \\
\cline { 2 - 3 } & Accuracy (\%) & Recall (\%) \\
\hline DL Network [12] & 91 & 73 \\
\hline VGG-16 [13] & 89 & 91 \\
\hline CheXNet [14] & 63 & 57 \\
\hline CADx [15] & 91 & 76 \\
\hline Proposed Method & $\mathbf{9 2}$ & $\mathbf{9 1}$ \\
\hline
\end{tabular}

\section{CONCLUSION}

In this paper, we have proposed an automatic algorithm for lung nodule recognition and classification. To begin with, we utilized the wiener filter to remove the undesired noise from the CXR images. and enhanced the images using adaptive histogram equalisation. Secondly, we performed classification on CXR images using simplified VGG framework. Finally, we have introduced an interface which will be developed in our future works so that our proposed algorithm can be used by the physicians in understanding and diagnosing the intensity of lung cancers. To prove the novelty of our proposed work we have compared our work with the latest state-of-art works as shown in Table I. The proposed method achieved 91\% sensitivity and $92 \%$ accuracy on training subset and accuracy of $94 \%$ on validation subset.

\section{ACKNOWLEDGMENT}

The authors of this paper acknowledges Department of Electronics and Electrical Engineering of Indian Institute of Technology Guwahati for supporting us through this research work.

\section{REFERENCES}

[1] W. Röntgen (Rontgen), "Über eine neue art von strahlen", Sitzungsberichte der Physikalisch-Medicinisch Gesellschaft zu Würzburg, pp. 132-141, 1895.

[2] H.-C. Shin et al., "Learning to read chest X-rays: Recurrent neural cascade model for automated image annotation", Proceedings of the IEEE Conf. on Comp. Vis. and Pat. Recog., pp. 2497-2506, 2016.

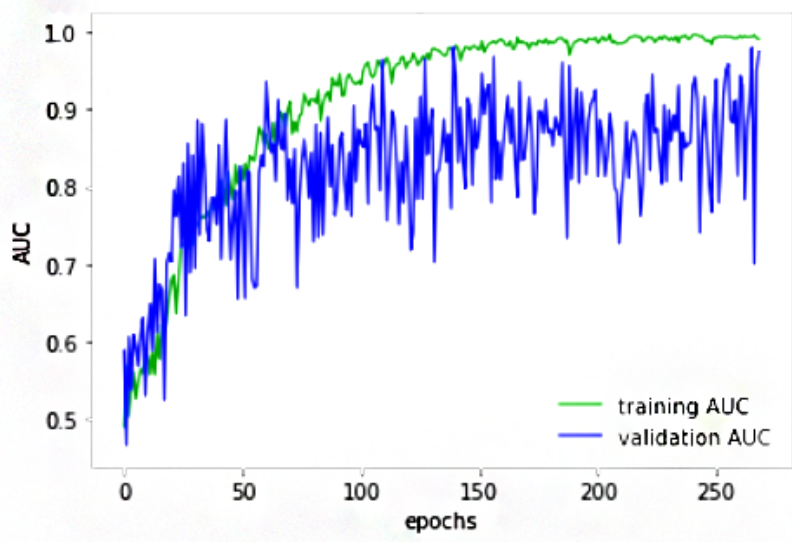

Fig. 8. Area Under Curve (AUC) for VGG network

[3] Z. Li et al., "Thoracic disease identification and localization with limited supervision", Proceedings of the IEEE Conf. on Comp. Vis. and Pat. Recog., pp. 8290-8299, 2018.

[4] F. Pasa et al., 'Efficient Deep Network Architectures for Fast Chest Xray Tuberculosis Screening and Visualization", Sci. Rep., vol. 9, no. 1, pp. $6268,2019$.

[5] X. Wang et al., "ChestX-ray8: Hospital-scale Chest X-ray Database and Benchmarks on Weakly-Supervised Classification and Localization of Common Thorax Diseases", May 2017.

[6] J. Irvin et al., "CheXpert: A Large Chest Radiograph Dataset with Uncertainty Labels and Expert Comparison", Jan 2019.

[7] A. G. Taylor, C. Mielke and J. Mongan, "Automated detection of moderate and large pneumothorax on frontal chest X-rays using deep convolutional neural networks: A retrospective study", PLOS Medicine, vol. 15, no. 11, pp. e1002697, 2018.

[8] L. Yao et al., "Learning to diagnose from scratch by exploiting dependencies among labels", Oct 2017.

[9] J. R. Zech et al., "Variable generalization performance of a deep learning model to detect pneumonia in chest radiographs: A crosssectional study", PLoS Med., vol. 15, no. 11, pp. e1002683, Nov. 2018.

[10] http://db.jsrt.or.jp/eng.php

[11] S. Trambadia and P. Dholakia, "Design and analysis of an image restoration using wiener filter with a quality based hybrid algorithms," 2015 2nd International Conference on Electronics and Communication Systems (ICECS), Coimbatore, 2015, pp. 1318-1323, doi: 10.1109/ECS.2015.7124798.

[12] Majdi, M. S., Salman, K. N., Morris, M. F., Merchant, N. C., \& Rodriguez, J. J. (2020). Deep Learning Classification of Chest X-Ray Images. In 2020 IEEE Southwest Symposium on Image Analysis and Interpretation, SSIAI 2020 - Proceedings (pp. 116-119).

[13] A. Siamak, R. Sadeghian, I. Abdellatif and S. Nwoji, \&quot;Diagnosing Heart Disease Types from Chest X- Rays Using a Deep Learning Approach,\&quot; 2019 International Conference on Computational Science and Computational Intelligence (CSCI), Las Vegas, NV, USA, 2019, pp. 910-913, doi: 10.1109/CSCI49370.2019.00173.

[14] P Rajpurkar, J Irvin, K Zhu et al., Chexnet: Radiologist-level pneumonia detection on chest X-rays with deep learning [J], 2017.

[15] L. Utkin, A. Meldo, M. Kovalev and E. Kasimov, \&quot; An Ensemble of Triplet Neural Networks for Differential Diagnostics of Lung Cancer,\&quot; 2019 25th Conference of Open Innovations Association (FRUCT), Helsinki, Finland, 2019, pp. 346-352, doi: 10.23919/FRUCT48121.2019.8981542. 